\title{
Multi-Objective Optimization of Traffic Signal Timing for Oversaturated Intersection
}

\author{
Yan Li, Lijie Yu, Siran Tao, and Kuanmin Chen \\ School of Highway, Chang'an University, The Middle Section of the Second Ring Road (South Part), Xian 710064, China \\ Correspondence should be addressed to Yan Li; lyan@chd.edu.cn
}

Received 31 August 2013; Revised 17 November 2013; Accepted 2 December 2013

Academic Editor: Wuhong Wang

Copyright (C) 2013 Yan Li et al. This is an open access article distributed under the Creative Commons Attribution License, which permits unrestricted use, distribution, and reproduction in any medium, provided the original work is properly cited.

For the purpose of improving the efficiency of traffic signal control for isolate intersection under oversaturated conditions, a multiobjective optimization algorithm for traffic signal control is proposed. Throughput maximum and average queue ratio minimum are selected as the optimization objectives of the traffic signal control under oversaturated condition. A simulation environment using VISSIM SCAPI was utilized to evaluate the convergence and the optimization results under various settings and traffic conditions. It is written by C++/CRL to connect the simulation software VISSIM and the proposed algorithm. The simulation results indicated that the signal timing plan generated by the proposed algorithm has good efficiency in managing the traffic flow at oversaturated intersection than the commonly utilized signal timing optimization software Synchro. The update frequency applied in the simulation environment was $120 \mathrm{~s}$, and it can meet the requirements of signal timing plan update in real filed. Thus, the proposed algorithm has the capability of searching Pareto front of the multi-objective problem domain under both normal condition and over-saturated condition.

\section{Introduction}

Current traffic signal control technologies usually have lower efficiency when the saturation degree is high. Many methods were proposed to improve the efficiency of traffic signal under traffic conditions with high saturation degree [1-3]. However, very little of those methods can be widely utilized for the reason of the requirements or limitations of those methods [4]. In order to establish one specific traffic control method with the capability of dealing with oversaturated condition, the nature of traffic signal control should be discovered.

Traffic signal control methods try to establish the connection between observed traffic parameters, like counts, delay and queue length, with traffic signal parameters, such as phase sequence, cycle length, and split. In this way, various traffic signal optimization algorithms can seek for the values of traffic signal parameters to obtain the optimal values of one or several traffic parameters by considering traffic signal parameters as independent variables under specific traffic condition. This principle is followed by almost all the commonly used traffic control optimization methods, which include TRRL (Transport and Road Research Laboratory) method [5], HCM (Highway Capacity Manual) method [6] and adaptive traffic control software like SCATS (Sydney Coordinated Adaptive Traffic System) [7] and SCOOT (Split Cycle Offset Optimizing Technique) [8]. Then, it can be indicated that the descriptions of traffic flow characteristics are the most critical factor in traffic signal timing optimization. However, the traffic flow becomes unstable when the traffic demand approaches or exceeds the capacity [9]. The detrimental effects, such as spillback, residual queue, or Defacto red, make it hard to describe traffic flow characteristics accurately. Thus, traffic signal optimization methods established by traffic flow formulas under normal traffic condition are no longer suitable for oversaturated conditions. The data-driven based heuristic algorithms could be an ideal method to obtain optimized traffic signal timing plan under oversaturated conditions. The heuristic algorithms do not rely on the traffic flow formulas but to seek for optimization scenarios based on real time traffic data. For the reason that various factors can affect the effects of traffic signal control under oversaturated condition, it will be better to consider more impact factors in the process of traffic signal optimization.

The focus of this paper is to present a multi-objective optimization method to obtain a relatively better signal 


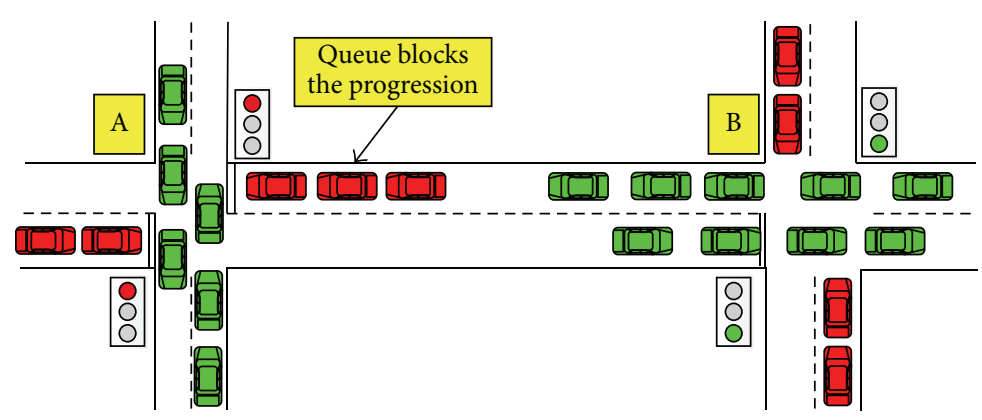

FIGURE 1: Residual queues at intersection.

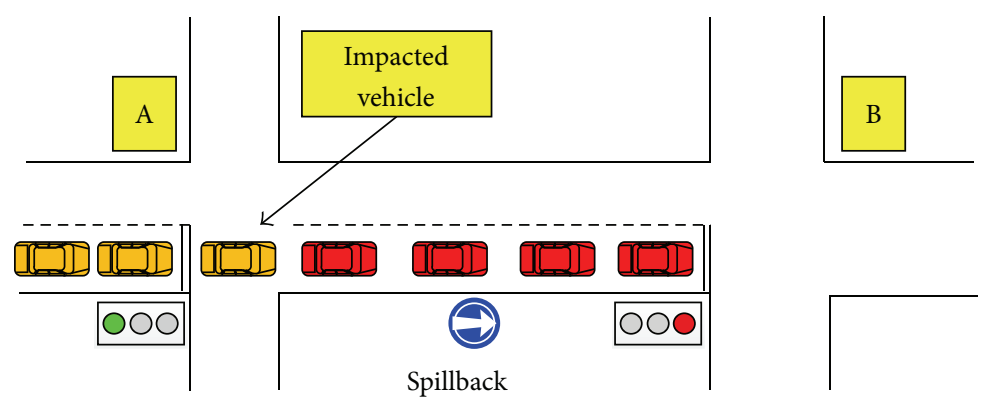

FIgURE 2: Spillback at intersection.

timing plan for oversaturated intersection. Before we design the algorithm, the characteristics of oversaturated traffic flow are analyzed to acquire the optimized objectives of the algorithm. Then, we present the details of the algorithm, which include coding scheme, optimization objectives, and algorithm selection. At last the convergence and simulation results of the algorithm under different conditions are summarized and analyzed.

\section{Characteristics of Oversaturated Flow}

The traffic status can be determined by traffic intensity, that is, the $\mathrm{V} / \mathrm{C}$ ratio. At signalized intersection, the over-saturation can be defined as the condition of an approach with residual queue [10], which is illustrated as Figure 1. As it is difficult to measure the residual queue directly, it can be estimated by loop detector [11] or mobile sensors [12] information using shockwave theory.

The traffic flow characteristics will be different when traffic flow is under over-saturation conditions, or approaching saturation conditions [13]. When the traffic condition is approaching saturation, the traffic flow will become unstable. A small fluctuation from any vehicle in a platoon may cause adverse consequences and reduce the efficiency of traffic system sharply [14]. When the traffic status is under oversaturated condition, the deterministic queuing model can be adopted to estimate the queue length. For the reason of low stability of saturated traffic flow, the parameters estimated by the models listed above can hardly be utilized in the process of signal timing.

Generally, oversaturated condition will firstly appear at one or several isolated intersection with relatively high saturation degree. Then, the congestion begins to spread to adjacent intersections with detrimental effect like "spillback," which is shown in Figure 2. Intersections along a route with larger traffic volume may spread the congestion much faster than other routes [15]. Finally, the whole road network will be congested, even in a "lock-out" status [16]. According to process of traffic congestion generation, the traffic control strategies under oversaturated condition should be utilized at isolated intersections to avoid detrimental effects like spillback or residual queue.

\section{Algorithm Selection and Design}

3.1. Selection of the Algorithm. Throughput maximum and queue ratio maintenance are two conflicting objectives, which is a typical multi-objective optimization problem (MOP) [17]. Many intelligent algorithms, such as Evolutionary Algorithm (EA) [18], Particle Swarm Optimization (PSO) [19], Simulated Annealing Algorithm (SA) [20], and Ant Colony Optimization (ACO) [21], can be utilized to obtain the Pareto front of the MOP. However, of all the algorithms, only the Genetic Algorithms (GA), one category of the Evolutionary Algorithm, was successfully applied in the traffic signal control system for commercial productions [22]. A Nondominated Sorting Genetic Algorithm II (NSGA-II) [23] has better performance than other multi-objective evolutionary algorithms [24], and thus it is selected as the traffic signal control optimization method in this paper.

The NSGA-II algorithm improved the NSGA by adding elitist strategy, density estimation strategy, and fast nondominated sorting strategy. The time complexity of NSGAII drops to $O\left(r N^{2}\right)$ from $O\left(r N^{3}\right)$ of NSGA, which is not 


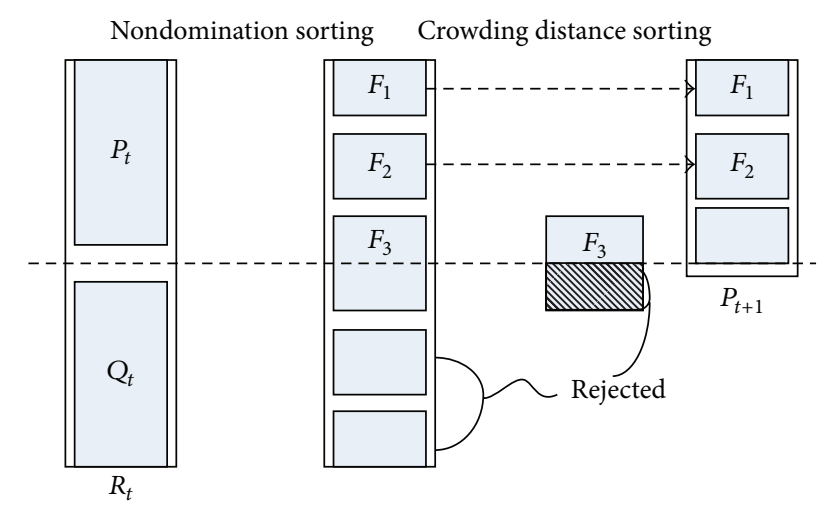

FIGURE 3: Major procedure of NSGA-II algorithm.

larger than other MOEA. The non-dominated sorting and the elitist strategy can improve the performance and keep the better solution. The density estimation strategy avoids the share parameter, which is hard to determine. These strategies make the NSGA-II algorithm have better performance than other MOEA. However, the convergence and diversity of the NSGA-II may be worse for the reason of density estimation strategy.

3.2. NSGA-II Algorithm. The major procedure of NSGA-II algorithm is shown in Figure 3. The main loop of the NSGA-II algorithm is listed as the following steps.

Step 1. Initially, a random parent population $P_{0}$ is created. The population is sorted based on the nondomination. Each solution is assigned a fitness (or rank) equal to its nondomination level. The usual binary tournament selection, recombination, and mutation operators are used to create an offspring population $Q_{0}$ of size $N$. Let $t=0$.

Step 2. A combined population $R_{t}=P_{t} \cup Q_{t}$ is formed. The population $R_{t}$ is of size $2 N$. Then, the population $R_{t}$ is sorted according to nondomination. The best non-dominated set $F_{i}$ is formed.

Step 3. The crowded-comparison operator $\prec_{n}$ is chosen to sort the non-dominated set $F_{i}$ in descending order. The best $N$ members of the set are chosen for the new population $P_{t+1}$.

Step 4. The new population $P_{t+1}$ is now used for selection, crossover and mutation to create a new population $Q_{t+1}$.

Step 5. When the termination condition meets, the loop stops; otherwise, $t=t+1$, and turn to Step 2 .

3.3. Coding Scheme. The coding scheme has evidently effects on genetic manipulation, especially for crossover. The binary encoding, real number encoding and structural encoding are three mostly used coding scheme in genetic algorithms. In the traffic signal control optimization problem, the coding scheme should get the capability to describe the signal timing plan. If the binary encoding is applied, additional constraints are needed in the process of crossover and mutation. Besides, the logical constraints between genes should also be considered (like the cycle length should be the summation of all the green times and lost times). All these constraints increase the complexity of the calculation. Hence, the real number encoding was selected as the coding scheme in traffic signal control optimization problem. The green time of each phase is the executable genetic operator of gene.

3.4. Selection of Optimization Objectives. Most practitioners tend to make simple changes to splits or phase sequence to minimize delay before moving to more complex approaches. Considering the traffic control strategies for oversaturated conditions are immature, it is better to keep the current control strategy instead of applying a new one. It is also important to implement strategies (where possible) to prevent over-saturation from occurring in the first place, rather than reacting to the issues after the fact. Once the traffic demand goes back to the normal level, the frequently used traffic control strategy will make the practitioners manage the traffic flow easily. In this way, the throughput maximum should be selected as one of the optimization objectives.

The queue problem is the major detrimental effects under oversaturated condition. Signal timing optimization algorithm should get the capability of maintaining the queue ratio of each approach in an acceptable range. In this control strategy, the green times should be adjusted to balance the queues at intersection's approaches during oversaturated conditions. The objective is to minimize the number of blocked intersections by critical intersection's queues.

Based on the discussion listed above, two optimization objectives, "throughput maximum" and "queue ratio maintenance," are selected in the proposed traffic signal timing optimization algorithm.

3.5. Optimization Objective Functions. In order to achieve the optimization objective of throughput maximum, the algorithm tends to extend cycle length to avoid the lost time brought by phase transition process. However, the queue of the conflicted intersection approaches will form very quickly under oversaturated condition and get extremely high probability to be "spillback" if the long cycle length is selected. At this time, the proposed algorithm should have the capabilities of maintaining the queue ratio at each approach to prevent the detrimental effects. It is assumed that high resolution traffic data are available for signal timing plan optimization. The output of the algorithm will be the green times of all the phases. The phase sequence is predefined.

Under oversaturated condition, traffic system is erratic, especially at the critical route of subnetwork or critical movement of the intersection. Hence, the primary target of traffic control under oversaturated condition is to maintain the traffic flow in a stable state and to discharge as many vehicles as possible, especially for the critical movement. For the purpose of maximizing the throughput vehicles of the critical movement, a weight coefficient should be multiplied by the actual throughput numbers in the corresponding optimization objective function. Thus, the throughput maximum 
optimization function in the algorithm can be obtained as follows:

$$
O_{c}=\max \sum_{i=1}\left(\omega_{i} \cdot n_{i}\left(g_{i}, C, t\right)\right)
$$

where $O_{c}$ is the weighted maximum throughput vehicle number of the intersection, $\omega_{i}$ is the weight coefficient of the $i$ th movement, and $n_{i}\left(g_{i}, C, t\right)$ is the actual throughput vehicles of the $i$ th movement, which is a function of the green time of movement $g_{i}$, cycle length $C$, and time; this parameter can be directly obtained from the VISSIM simulation environment in this research.

Many traffic optimization measures can be classified as the queue maintenance strategy, such as average queue length, maximum queue length, average maximum queue length and dissipated queue length. The queue occupancy ratio is utilized as the optimization objective by considering the impact of acceptable queue space. By introducing this conception in the optimization objective function, the detrimental effect "spillback" can be eased effectively. Meantime, the queue length of each phase can also be optimized. The queue ratio maintenance optimization function in the algorithm can be obtained as follows:

$$
\mathrm{O}_{l}=\min \frac{1}{p} \sum_{p=1}^{P} \frac{\operatorname{len}_{p}}{L_{p}}
$$

where $p$ is the number of the phases, $\operatorname{len}_{p}(t)$ is the queue length of the $p$ th phase, and $L_{p}$ is the maximum acceptable queue of the corresponding phase.

\section{Evaluation Setup}

4.1. Simulation Environment. The proposed algorithm was deployed and evaluated in a prevailing microscopic traffic simulation environment, VISSIM. The advantages of VISSIM over other simulation packages include the following

(1) VISSIM provides the largest flexibility for users to calibrate driving behaviors and traffic conditions.

(2) VISSIM was developed under .NET framework, which brings flexibility for add-on program development.

(3) VISSIM provides the best tools for the development of signal control strategies, such as the NEMA controller emulator, Vehicle Actuated Programming (VAP) language, signal control Application Programming Interfaces (SCAPI), and so forth.

VISSIM SCAPI method was used to develop the signal control emulator in this research. SCAPIs were written in $\mathrm{C}++$ /CLR language [25] and the original version of SCAPI controller requires signal control algorithms to be embedded into a single dynamic link library (DLL) file. To facilitate the development, a middleware was developed, which can synchronously collect all the real-time detectors/phases states from the VISSIM network to the controller emulator then

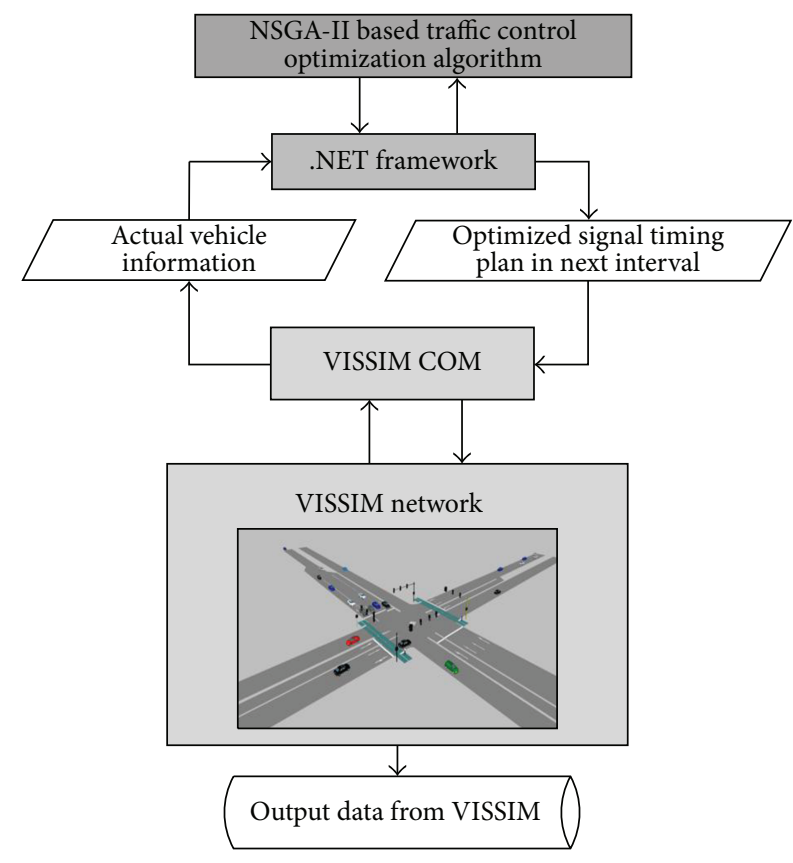

FIGURE 4: Simulation environment of VISSIM.

return the new desired phase states back to the VISSIM network. At each time step, the controller runs the algorithm, makes decisions according to the current state, and then returns the new desired phase states to the VISSIM network. The concept of this simulation environment is illustrated as in Figure 4.

The algorithm was tested under a PC environment with the configuration of Intel Core i7-3770 3.4 GHz processor and 8 GB DDR3 RAM. It takes less than $1 \mathrm{~s}$ to optimize the signal timing plan of single intersection on average. The VISSIM software has the capability of simulating the network ten times faster than the real time at minimum. Thus, a $120 \mathrm{~s}$ interval, which is larger than most cycle length applied in the real filed, is selected as the update frequency. This parameter should be adjusted based on the hardware configuration. During the period of updating the signal control parameters, the simulation software can provide the simulated traffic flow parameters in the next 20 minutes, which can become the input of the algorithm. The signal timing plan will be updated synchronously in the simulation environment when the current cycle is finished.

4.2. Experimental Design. A simple four way intersection is selected to evaluate the proposed algorithm. The geometric characteristics of the intersection are shown in Figure 5. The experimental configurations are listed in Table 1, and experimental traffic volume is listed in Table 2. Within the signal timing plan, the NEMA's (National Electrical Manufactures Association) ring structure (see Figure 7) [26] is utilized to achieve the purpose of adjusting the green flexibly based on traffic volume. 
TABLE 1: Experimental configurations of the proposed algorithm.

\begin{tabular}{lc}
\hline Parameters & Desired value \\
\hline The length of intersection approach $(\mathrm{m})$ & 550 \\
Desired speed $(\mathrm{km} / \mathrm{h})$ & 60 \\
Number of genes & 4 for signal ring \\
& 5 for signal ring and phase sequence \\
Population size & 8 for dual-rings \\
Crossover probability & 200 \\
Mutation probability & 0.8 \\
Evolution generation & 0.1 \\
\hline
\end{tabular}

TABLE 2: Experimental traffic volume (pcu).

\begin{tabular}{lccccccc}
\hline \multirow{2}{*}{ Movement } & \multicolumn{2}{c}{ Eastbound } & \multicolumn{2}{c}{ Westbound } & \multicolumn{2}{c}{ Northbound } & \multicolumn{2}{c}{ Southbound } \\
& Low & High & Low & High & Low & High & Low \\
\hline Left turn & 65 & 130 & 30 & 60 & 30 & 60 & 40 \\
Throughput & 620 & 1240 & 700 & 1400 & 370 & 740 & 80 \\
Right turn & 35 & 70 & 20 & 60 & 20 & 40 & 510 \\
\hline
\end{tabular}

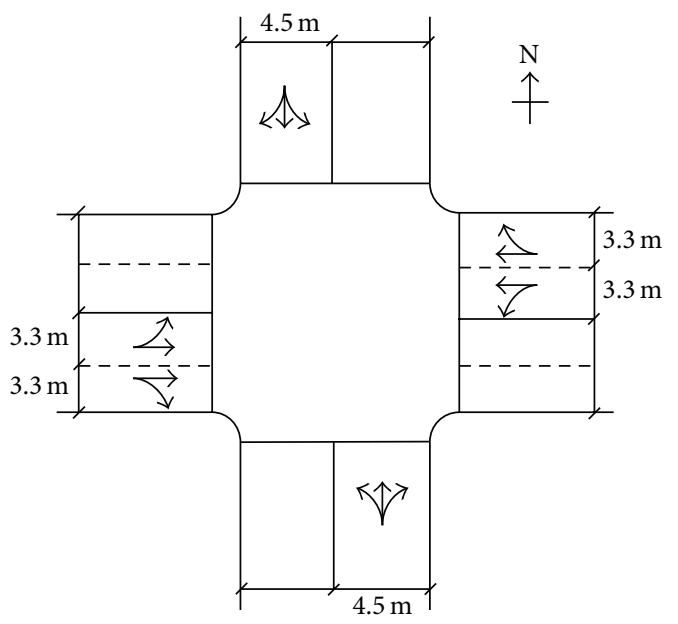

FIGURE 5: Intersection geometry.

\section{Results and Discussions}

The convergence and results of proposed algorithm at various gene numbers and traffic volumes are analyzed. In order to evaluate the effect of the algorithm, the widely used Webster model and Synchro software are utilized as a reference algorithm to the proposed algorithm.

5.1. Convergence of the Algorithm. In order to test the convergence of the algorithm, the optimization results of the proposed algorithm after 50 generations and 100 generations are compared. As illustrated in Figure 6, the converged speed of the proposed algorithm is relatively fast. After 50 generations, the optimization results have already got a relatively good convergence, which is similar to the optimization results after 100 generations. In this way, the evaluation generation can be reduced when it is necessary to consider the calculation time. Although the accuracy of the solution may be lower, the accuracy can still be acceptable based on the experimental results.

5.2. Optimization Results with Different Gene Numbers. As the NEMA's ring structure is utilized in the proposed algorithm, the number of genes can stand for different signal timing scenarios. In this paper, a comparison of scenarios with 4,5 , and 8 genes is selected. The scenario with 4 genes stands for a signal ring timing plan with fixed four phases (left lagging). While the scenario with 5 genes means a signal ring with flexible phase sequence (lead/lagging). And the 8 genes scenario is a standard NAME dual-ring traffic signal timing plan. Figure 7 shows the single ring structure with left lag phase sequence and the standard dual ring structure. Figure 8 illustrates the optimization results of the three scenarios listed above. It can be indicated that the feasible optimal solutions of the 4 and 5 genes are much more than the 8 genes scenario. The optimization results will be better if the distribution of optimal solution can cover the whole period. The distribution of the optimization solutions of 5 genes are more balanced than other scenarios. With the increase of the gene's number, the evaluation generations and population size will rise significantly. Meantime, the complexity of the algorithm will increase too.

5.3. Comparison of the Optimization Results under Different Traffic Volume. As illustrated in Figure 9, the proposed algorithm has the capability of optimizing traffic signal control under different traffic conditions. In the high volume scenario, the traffic volume of southbound exceeds its 


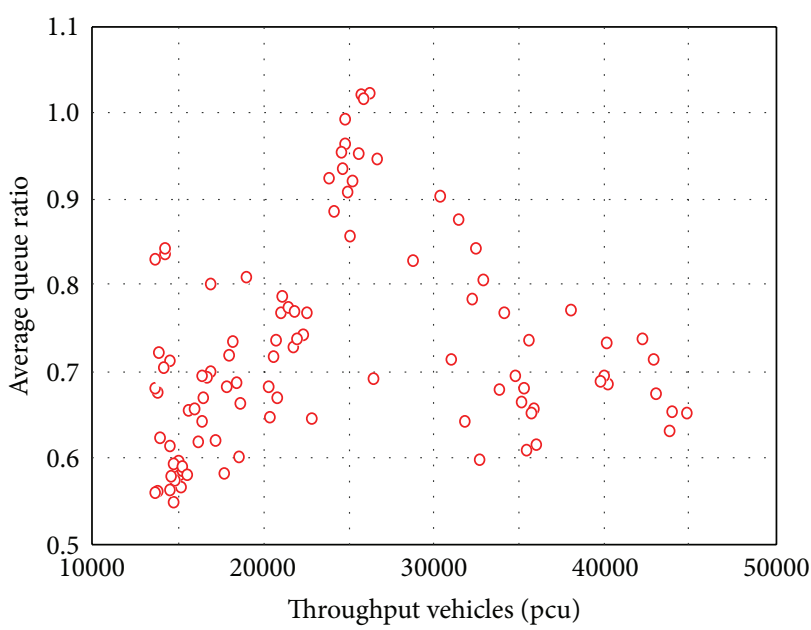

(a) Initial distribution

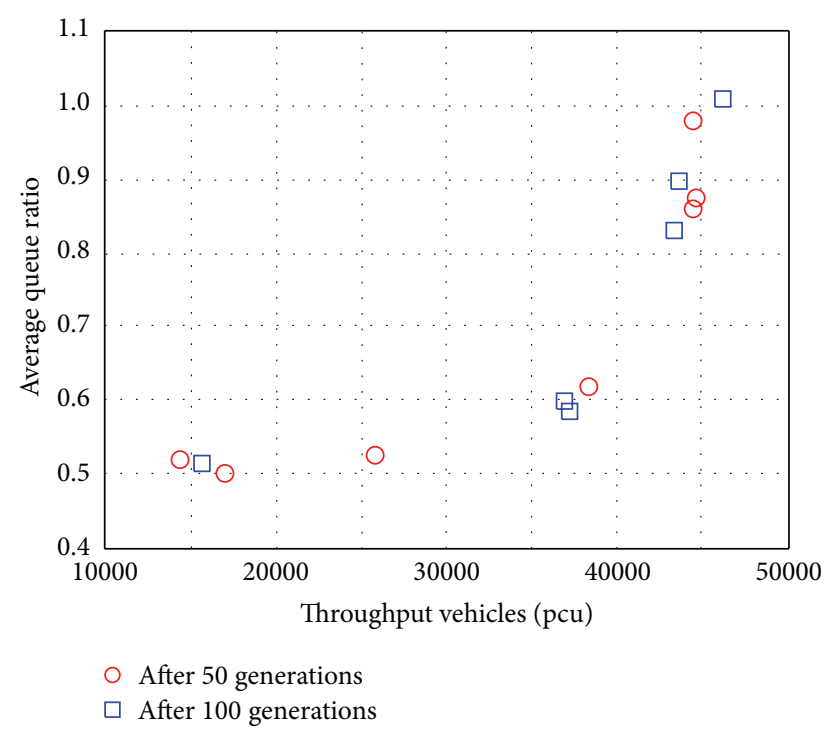

(b) Distribution of optimal solutions after 50 and 100 generations

FIGURE 6: Distribution of optimal solutions after various generations.

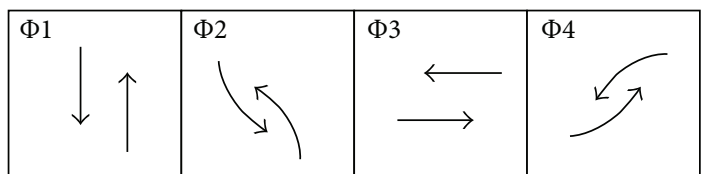

(a) Single ring structure

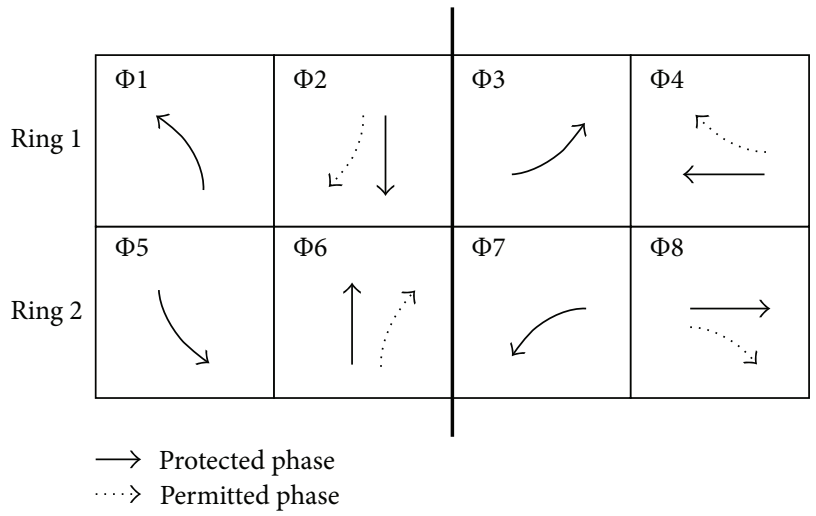

(b) Standard NAME dual-ring structure

FIGURE 7: Schematic diagrams of the ring structure.

capacity. The selected intersection is under an oversaturated condition. The optimization results of the Webster method and Synchro are shown in Table 3, and the comparisons of the selected parameters from the methods are shown in Table 4. The Webster model cannot calculate the cycle length with a saturation degree greater than 1; thus the Akcelik's model is applied to obtain the signal timing plan under oversaturated condition. Based on the optimal results, the average queue ratio of the intersection can be maintained less than 1 by applying the proposed algorithm.

Compared with the optimal results with Webster model and Synchro software, the proposed algorithm has similar performance with other method under normal condition. However, under oversaturated condition, queues build up quickly under the signal timing plan by common used models. For the reason of applying specific optimization objectives, the proposed algorithm has the capability of searching and converging to Pareto front of the multiobjective problem domain under oversaturated conditions.

It should be noticed that the proposed algorithm has no obvious advantages under the normal conditions. In this way, it is suggested to apply the mature signal timing optimization methods or software, such as Synchro, to determine the signal timing plan under the normal conditions instead of applying the new one. It is also important to implement the proposed algorithm to prevent over-saturation from occurring in the first place, rather than reacting to the issues after the fact. Once the traffic demand goes back to the normal level, the frequently used traffic control method will make the practitioners manage the traffic flow easily.

\section{Conclusions}

A multi-objective optimization algorithm is present to optimize traffic signal timing at oversaturated intersection. In 


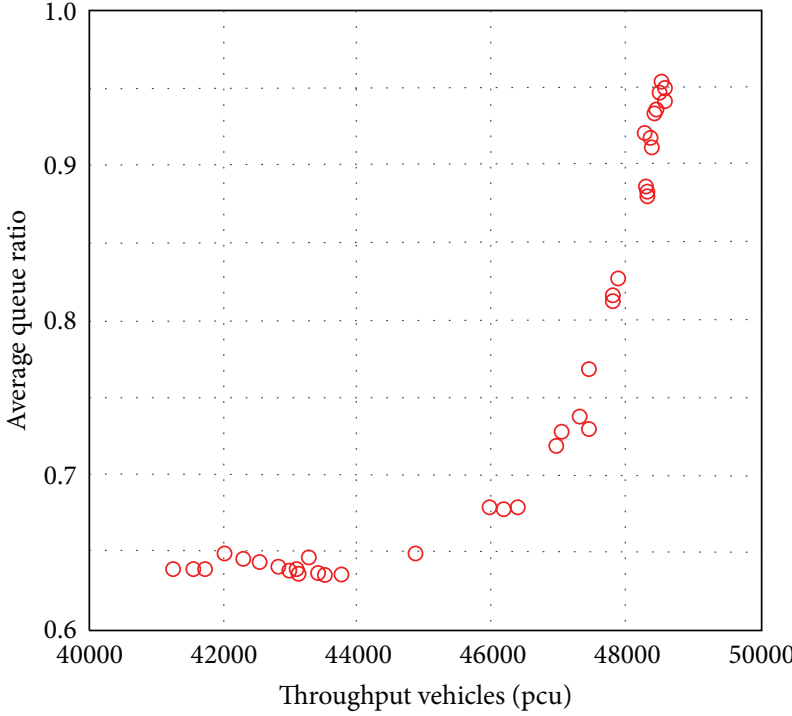

(a) 4 genes

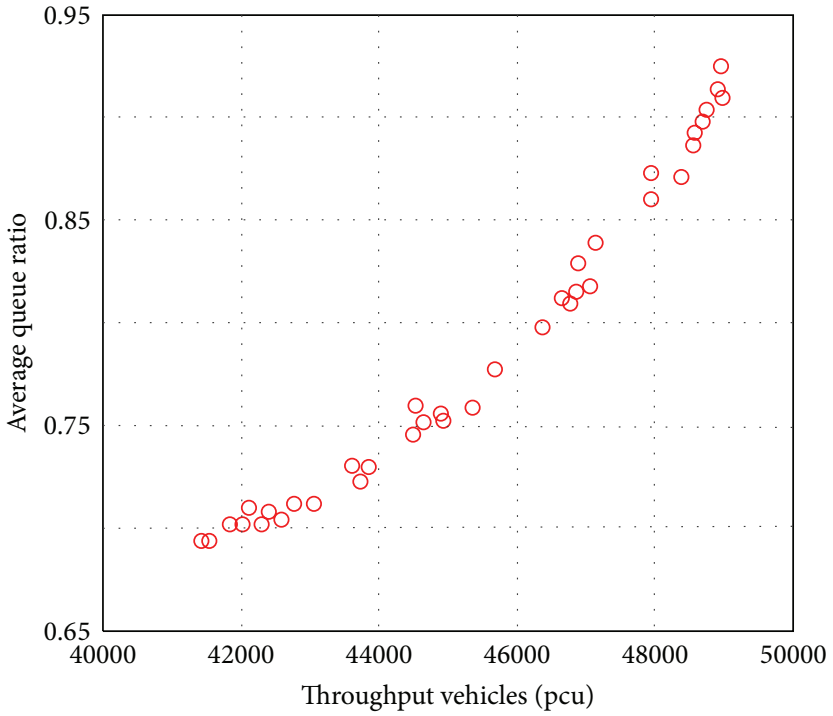

(b) 5 genes

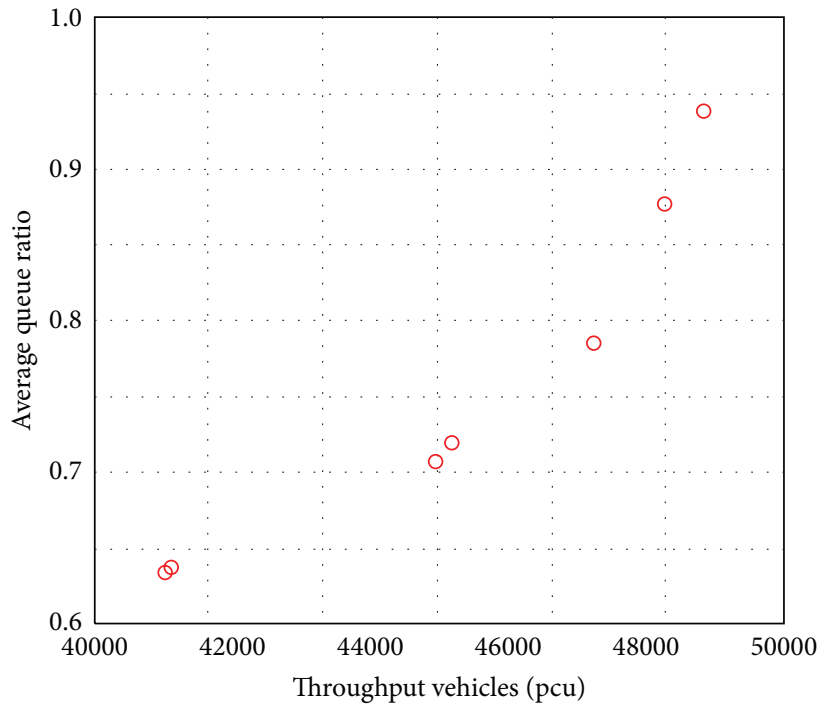

(c) 8 genes

FIGURE 8: Distribution of the optimal solutions with various gene numbers.

TABLE 3: Optimized signal timing plan of Webster model and Synchro software.

\begin{tabular}{lcccc}
\hline Algorithm & Cycle length (s) & Phase 1(s) & Phase 2(s) & Yellow (s) \\
\hline Webster model under normal condition & 66 & 35 & 23 & 4 \\
Webster model under over-saturated condition & 132 & 74 & 50 & 4 \\
Synchro model under normal condition & 70 & 36 & 45 & 37 \\
Synchro model under over-saturated condition & 90 & 45 & 4 \\
\hline
\end{tabular}

TABLE 4: Comparison of the evaluation results between proposed method and other models.

\begin{tabular}{|c|c|c|c|c|}
\hline \multirow{2}{*}{ Algorithm } & \multicolumn{2}{|c|}{ Throughput vehicles (pcu) } & \multicolumn{2}{|c|}{ Average queue ratio } \\
\hline & Normal & Over-saturated & Normal & Over-saturated \\
\hline NSGA II (4 genes) & 23417 & 44492 & 0.46 & 0.94 \\
\hline NSGA II (5 genes) & 24582 & 46706 & 0.44 & 0.94 \\
\hline NSGA II (8 genes) & 24814 & 48628 & 0.45 & 0.90 \\
\hline Webster & 23981 & 29598 & 0.48 & $>1$ \\
\hline Synchro & 24173 & 35916 & 0.45 & $>1$ \\
\hline
\end{tabular}




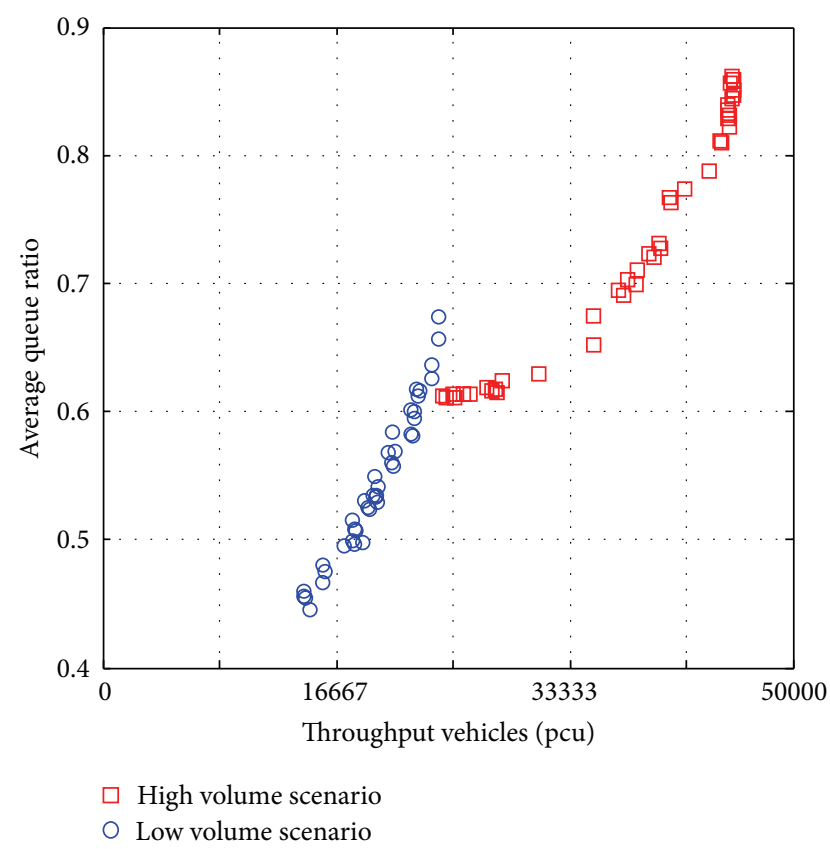

FIGURE 9: Distribution of the optimal solutions under different traffic volumes.

the proposed algorithm, throughput maximum and average queue ratio minimum are selected as the optimization objectives to meet the characteristics of oversaturated traffic flow. The proposed algorithm was tested under various kinds of phases and traffic conditions. The gene number can reflect the phase sequence and traffic control structure of the signal timing. The more genes are selected, the more accurate the algorithm is. Meantime, the proposed algorithm will be difficult to get feasible solution if the large gene number is selected because of the computational complexity. Thus, it is suggested that the gene number should be no more than 8. The real number encoding is utilized in the proposed algorithm, and the executable genetic operator of gene is the green time of the corresponding phase. The update frequency applied in the simulation environment was $120 \mathrm{~s}$, and it can meet the requirements of signal timing plan update in real filed. The experimental results from VISSIM SCAPI simulation environment indicate that the proposed algorithm has the ability of obtaining traffic signal timing plan with better performance than the commonly utilized signal timing optimization software under oversaturated conditions. The performance of proposed algorithm under normal condition is not better than the commonly used traffic signal control optimization methods for the reason of the discrepancies between the optimization objectives and the traffic flow characteristics. The proposed algorithm has the capability of searching Pareto front of the multi-objective problem domain. Further jobs should be concerned on the signal timing optimization method for oversaturated coordinated intersections or small scale road network and real field applications with the traffic signal controller.

\section{Acknowledgments}

This research was supported by the National Nature Science Foundation of China (no. 51208054) and the Fundamental Research Funds for the Central Universities (no. 2013G1211005).

\section{References}

[1] M. M. Abbas, Z. M. Adam, and D. Gettman, "Development and evaluation of optimal arterial control strategies for oversaturated conditions," Transportation Research Record, no. 2259, pp. 242-252, 2011.

[2] G. Abu-Lebdeh and R. F. Benekohal, "Design and evaluation of dynamic traffic management strategies for congested conditions," Transportation Research A, vol. 37, no. 2, pp. 109-127, 2003.

[3] G. Abu-Lebdeh and R. F. Benekohal, "Signal coordination and arterial capacity in oversaturated conditions," Transportation Research Record, no. 1727, pp. 68-76, 2000.

[4] Y. Li, X. Guo, J. Yang, Y. Liu, and S. He, "Mechanism analysis and implementation framework for traffic signal control of oversaturated intersection group," Journal of Transportation Systems Engineering and Information Technology, vol. 11, no. 4, pp. 2834, 2011.

[5] M. Papageorgiou, C. Diakaki, V. Dinopoulou, A. Kotsialos, and Y. Wang, "Review of road traffic control strategies," Proceedings of the IEEE, vol. 91, no. 12, pp. 2043-2067, 2003.

[6] Transportation Research Board, Highway Capacity Manual, National Academy of Sciences, Washington, DC, USA, 5th edition, 2010.

[7] A. G. Sim and K. W. Dobinson, "The Sydney coordinated adaptive traffic (SCAT) system philosophy and benefits," IEEE Transactions on Vehicular Technology, vol. 29, no. 2, pp. 130-137, 1980.

[8] R. D. Bretherton and G. T. Bowen, "Recent enhancements to SCOOT-SCOOT version 2.4," in Proceedings of the $3 \mathrm{rd}$ International Conference on Road Traffic Control, pp. 95-98, London, UK, May 1990.

[9] F. Dion, H. Rakha, and Y.-S. Kang, "Comparison of delay estimates at under-saturated and over-saturated pre-timed signalized intersections," Transportation Research B, vol. 38, no. 2, pp. 99-122, 2004.

[10] R. W. Denney, L. Head, and K. Spencer, Signal Timing under Saturated Conditions, Federal Highway Administration, Washington, DC, USA, 2008.

[11] X. Wu, H. X. Liu, and D. Gettman, "Identification of oversaturated intersections using high-resolution traffic signal data," Transportation Research C, vol. 18, no. 4, pp. 626-638, 2010.

[12] X. J. Ban, P. Hao, and Z. Sun, "Real time queue length estimation for signalized intersections using travel times from mobile sensors," Transportation Research C, vol. 19, no. 6, pp. 1133-1156, 2011.

[13] B. B. Park and C. Li, "An analytical approach for estimating the highway capacity manual signalized intersection delay variability," Computer-Aided Civil and Infrastructure Engineering, vol. 26, no. 4, pp. 327-333, 2011.

[14] F. Zheng and H. Van Zuylen, "Uncertainty and predictability of urban link travel time: delay distribution-based analysis," Transportation Research Record, no. 2192, pp. 136-146, 2010. 
[15] A. Chen, H. Yang, H. K. Lo, and W. H. Tang, "Capacity reliability of a road network: an assessment methodology and numerical results," Transportation Research B, vol. 36, no. 3, pp. 225-252, 2002.

[16] E. J. Smaglik, D. M. Bullock, D. Gettman, C. M. Day, and H. Premachandra, "Comparison of alternative real-time performance measures for measuring signal phase utilization and identifying oversaturation," Transportation Research Record, no. 2259, pp. 123-131, 2011.

[17] K. Ghoseiri, F. Szidarovszky, and M. J. Asgharpour, "A multiobjective train scheduling model and solution," Transportation Research B, vol. 38, no. 10, pp. 927-952, 2004.

[18] J.-H. Kim, J.-H. Han, Y.-H. Kim, S.-H. Choi, and E.-S. Kim, "Preference-based solution selection algorithm for evolutionary multiobjective optimization," IEEE Transactions on Evolutionary Computation, vol. 16, no. 1, pp. 20-34, 2012.

[19] J. Jie, J. Zeng, C. Han, and Q. Wang, "Knowledge-based cooperative particle swarm optimization," Applied Mathematics and Computation, vol. 205, no. 2, pp. 861-873, 2008.

[20] W.-C. Hong, "Traffic flow forecasting by seasonal SVR with chaotic simulated annealing algorithm," Neurocomputing, vol. 74, no. 12-13, pp. 2096-2107, 2011.

[21] R. Putha, L. Quadrifoglio, and E. Zechman, "Comparing ant colonyoptimization and genetic algorithm approaches for solving traffic signal coordination under oversaturation conditions," Computer-Aided Civil and Infrastructure Engineering, vol. 27, no. 1, pp. 14-28, 2012.

[22] B. Park, C. Messer, and T. Urbanik II, “Traffic signal optimization program for oversaturated conditions: genetic algorithm approach," Transportation Research Record, no. 1683, pp. 133142, 1999.

[23] K. Deb, A. Pratap, S. Agarwal, and T. Meyarivan, "A fast and elitist multiobjective genetic algorithm: NSGA-II," IEEE Transactions on Evolutionary Computation, vol. 6, no. 2, pp. 182197, 2002.

[24] D. Sun, R. F. Benekohal, and S. T. Waller, "Multiobjective traffic signal timing optimization using non-dominated sorting genetic algorithm," in Proceedings of IEEE Intelligent Vehicles Symposium, pp. 198-203, Columbus, Ohio, USA, 2003.

[25] D. Lacks and T. Kocak, "Developing reusable simulation core code for networking: the grid resource discovery example," Journal of Systems and Software, vol. 82, no. 1, pp. 89-100, 2009.

[26] National Electrical Manufactures Association, National Transportation Communication for ITS Protocol Object Definitions for Actuated Traffic Controller Units, NEMA, Washington, DC, USA, 1996, no. TS3.5, 1996. 


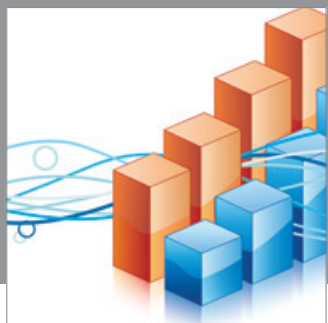

Advances in

Operations Research

mansans

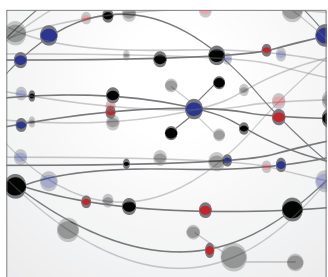

The Scientific World Journal
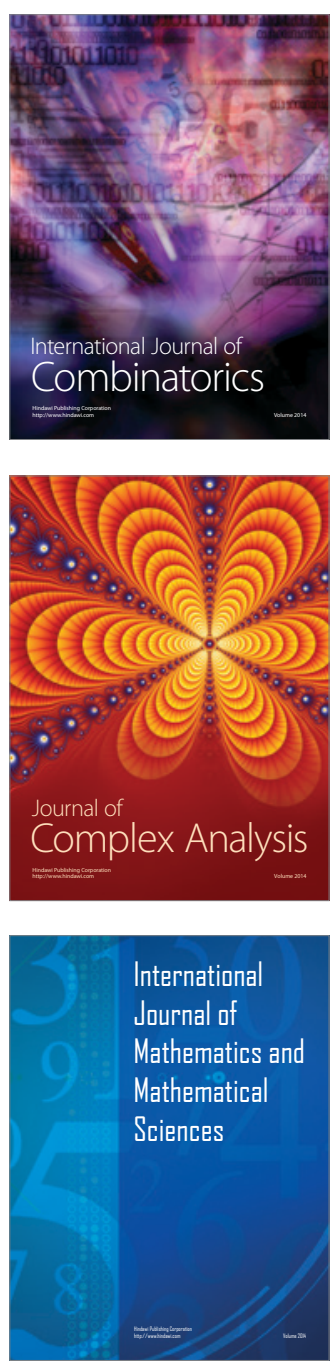
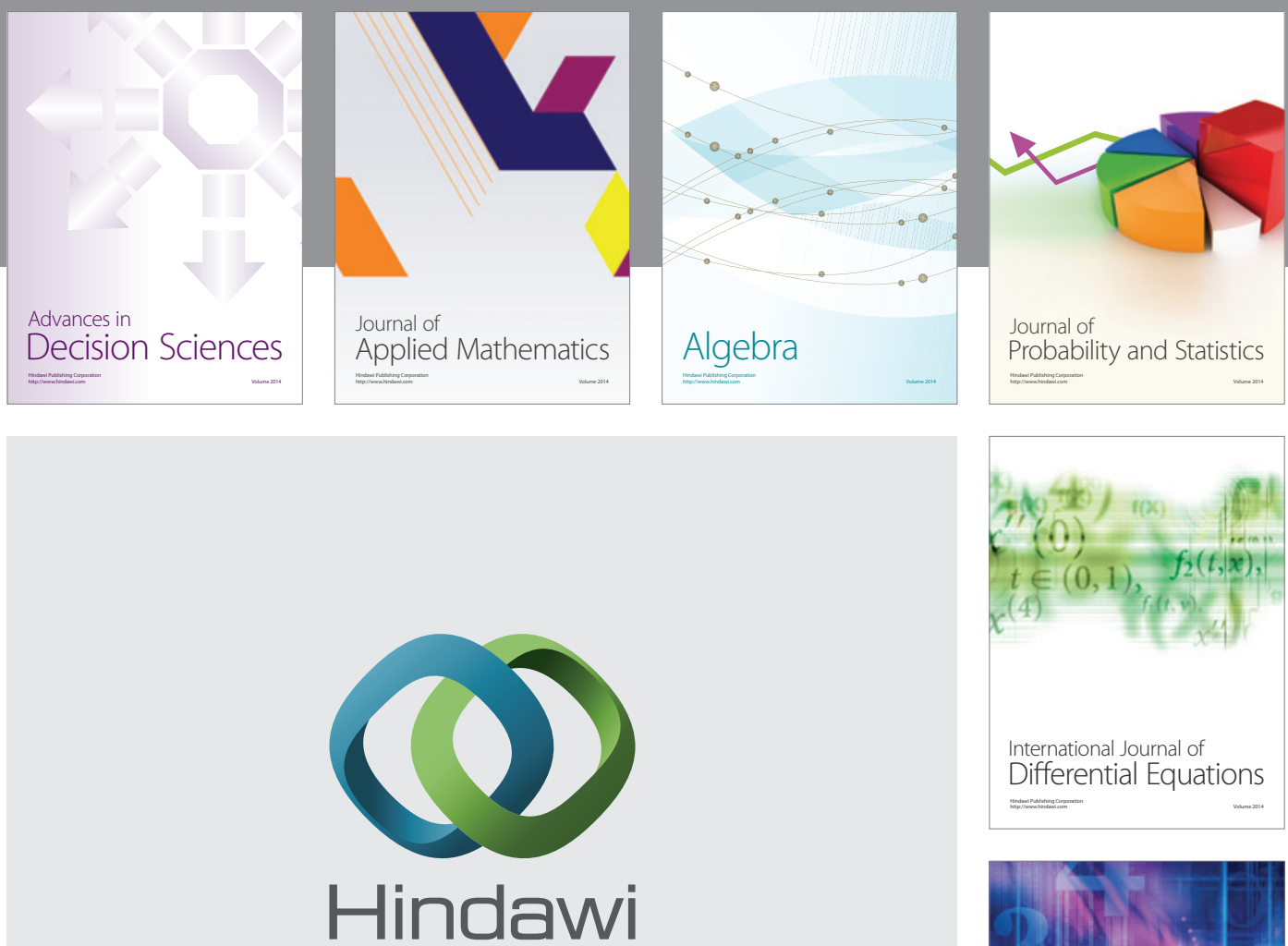

Submit your manuscripts at http://www.hindawi.com
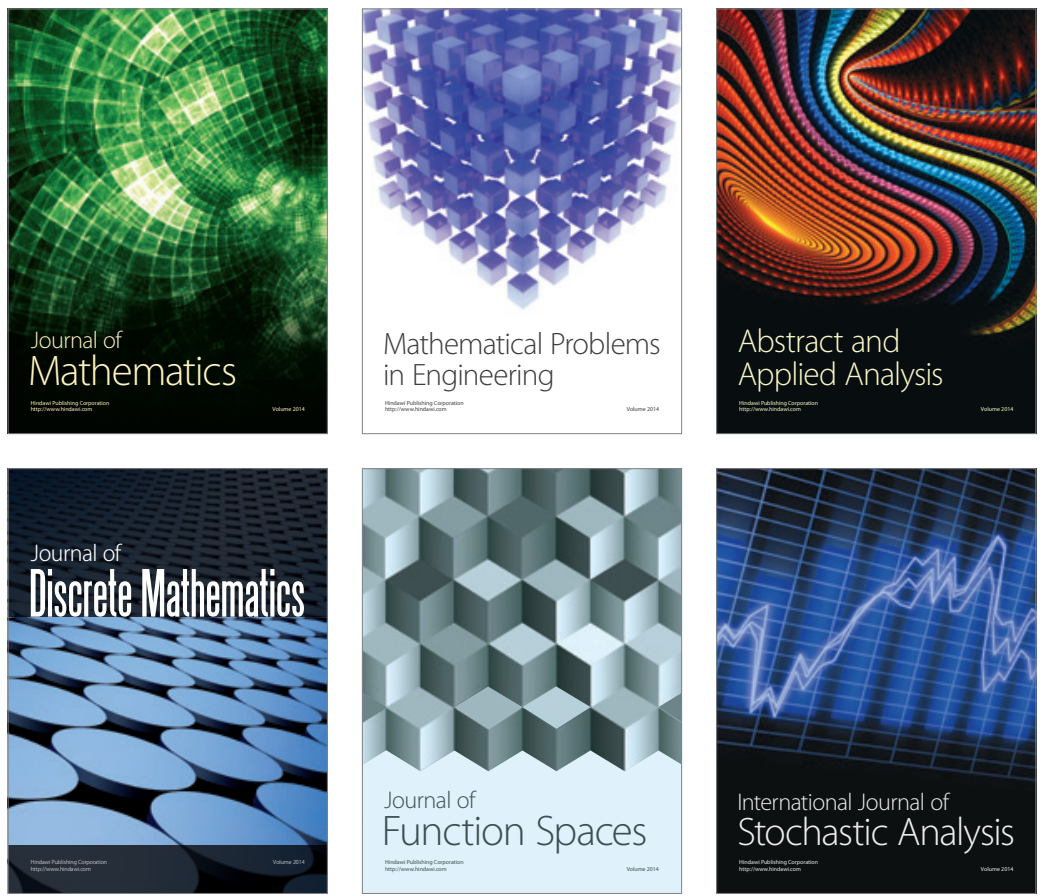

Journal of

Function Spaces

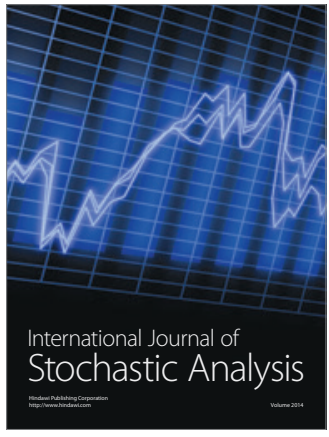

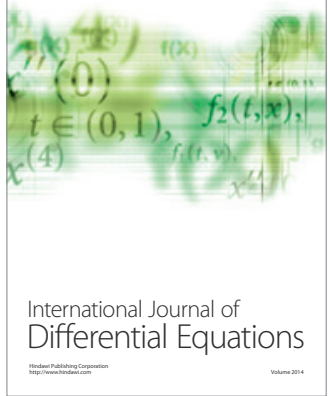
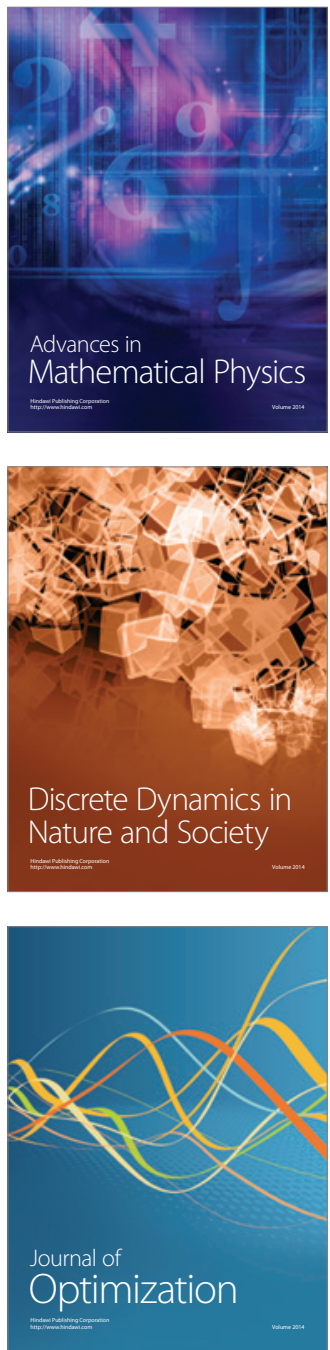